\title{
Prior statin therapy and mortality among critically ill patients: a systemic review and meta-analysis of cohort studies
}

\author{
Tak Kyu Oh ${ }^{1}$, In-Ae Song ${ }^{1}$, Sunkyu Choi $^{2}$ \\ ${ }^{1}$ Department of Anesthesiology and Pain Medicine, ${ }^{2}$ Medical Research Collaborating Center, Seoul National University Bundang Hospital, \\ Seongnam, Korea \\ Contributions: (I) Conception and design: TK Oh; (II) Administrative support: None; (III) Provision of study materials or patients: IA Song, S Choi; \\ (IV) Collection and assembly of data: IA Song, S Choi; (V) Data analysis and interpretation: TK Oh; (VI) Manuscript writing: All authors; (VII) Final \\ approval of manuscript: All authors. \\ Correspondence to: In-Ae Song. Department of Anesthesiology and Pain Medicine, Seoul National University Bundang Hospital, 166 Gumi-ro, \\ Bundang-gu, Seongnam 463-707, Korea. Email: songoficu@outlook.kr.
}

\begin{abstract}
The effect of prior statin exposure in critically ill patients remains controversial and has not been established in previous cohort studies. We performed a systematic review of previous cohort studies to evaluate the association of prior statin therapy with mortality in critically ill patients and conducted a meta-analysis. The MEDLINE, EMBASE, and Cochrane CENTRAL databases, from their inception to January 7, 2020, were used for this study. Statin users were defined as patients prescribed statin regularly before intensive care unit admission or diagnosis of a specific disease, such as sepsis. The Cochran chisquare test and I statistics were used to determine heterogeneity between studies. In total, 199,985 critically ill patients from nine studies (44,582 statin users and 155,403 non-statin users) were included in the metaanalysis. According to the random effect model, the 30-day mortality of statin users was $31 \%$ lower than that of non-statin users (hazard ratio: $0.69,95 \%$ confidence interval: 0.56 to 0.85 ). This association was similar in atorvastatin users and simvastatin users. However, hospital mortality in statin users was not significantly associated with that in non-statin users [odds ratios (ORs): $0.71,95 \%$ CI: 0.42 to 1.21 ]. This study showed that there was a beneficial association of prior statin therapy with 30-day mortality in critically ill patients. However, there was no significant association with hospital mortality. Additional prospective cohort studies with a large sample size should be performed to confirm these findings.
\end{abstract}

Keywords: Critical care; critical illness; hospital mortality; intensive care unit; meta-analysis

Submitted Nov 22, 2019. Accepted for publication Jan 17, 2020.

doi: 10.21037/atm.2020.02.101

View this article at: http://dx.doi.org/10.21037/atm.2020.02.101

\section{Introduction}

Statins are among the most commonly prescribed drugs for the treatment of dyslipidemia worldwide (1). They mainly lower the risk of acute cardiovascular events by lowering serum cholesterol levels (2). They also have anti-inflammatory, anti-thrombotic, and immunomodulating effects $(3,4)$. These effects are referred to as "pleiotropic effects" (5), which improve the outcomes of various diseases (6).

The effects of statin therapy in critically ill patients have been evaluated in many studies. Previous meta-analyses have shown the benefit of statin therapy in terms of mortality among patients with severe infection or sepsis $(7,8)$. In contrast, some randomized clinical trials (RCTs) have shown no benefit of statin therapy on mortality in patients with sepsis or septic shock (9-11), and this was supported by a recent meta-analysis (12). However, these RCTs focused on the effect of statin therapy after intensive care unit (ICU) admission or diagnosis of severe conditions such as sepsis or acute respiratory distress syndrome (ARDS). Although some cohort studies focused on the effect of statin therapy before 
ICU admission or diagnosis of a specific disease (e.g., sepsis or ARDS) on mortality in critically ill patients (13-21), their results remain controversial. Whereas some cohort studies reported a benefit of prior statin therapy on mortality among critically ill patients (13-19), others reported that there was no significant association in this regard $(20,21)$.

Therefore, we performed a systematic review of previous cohort studies to evaluate the association of prior statin therapy with mortality and performed a meta-analysis with a larger sample size than that in previous cohort studies.

\section{Methods}

\section{Search strategy}

This systematic review was performed in compliance with the Meta-analysis of Observational Studies in Epidemiology guidelines (22) and the Preferred Reporting Items for Systematic Reviews and Meta-Analyses guidelines [2009] (23). The MEDLINE, EMBASE, and Cochrane CENTRAL databases were searched for studies conducted on humans and published in English. The databases were searched, from their inception to January 7, 2020, for the following terms by TK Oh and IA Song: "Statin and Critically ill", "Statin and Intensive Care Units", "Statin and Sepsis", "Statin and ARDS", and "Preadmission Statin". The literature that included the search terms in the title, abstract, or keywords were initially searched, and articles that did not meet the eligibility criteria were excluded by TK Oh and IA Song after discussion. Any disagreement was resolved by $\mathrm{S}$ Choi after discussion.

\section{Eligibility criteria}

First, cohort studies were considered suitable for inclusion in this meta-analysis if they enrolled "critically ill patients" who required treatment or monitoring in the ICU. Therefore, mixed ICU patients, patients with sepsis or septic shock, ARDS patients, and patients with ventilatorassociated pneumonia (VAP) were included in the category of critically ill patients in this meta-analysis. Second, cohort studies were mainly included if they investigated the association between prior or preadmission statin therapy and mortality among critically ill patients. The 30-day mortality and in-hospital mortality were considered outcomes. If a study focused on only statin treatment after ICU admission or hospitalization and not prior statin therapy, then it was excluded from the meta-analysis.

\section{Selection of studies and data extraction}

We extracted data regarding the name of the first author, year of publication, country in which the research was conducted, study design (i.e., single or multicenter cohort study), study period, diagnosis of the study population (e.g., sepsis or ARDS), number of patients in each group, main statistical method (multivariable adjustment or propensity score matching), and endpoints. The primary endpoints for this meta-analysis were 30-day mortality or hospital mortality after ICU admission or diagnosis of a specific disease (sepsis or ARDS). Adjusted hazard ratios (HRs) or odds ratios (ORs) for primary endpoints after propensity score matching were considered for this meta-analysis if the cohort study used both propensity score matching and multivariable adjustment. In addition, 28-day mortality was considered as 30-day mortality for the purpose of this metaanalysis. Whereas a study conducted at a single institution was considered a single-center cohort study, a study using a national health database was considered a multicenter cohort study.

\section{Quality assessment of the studies}

The risk of bias for each outcome in all included studies was evaluated using the Newcastle Ottawa Scale (24) by TK $\mathrm{Oh}$ and IA Song, and any disagreement was resolved by S Choi after discussion. Studies that scored 7-9 points on the Newcastle Ottawa Scale were considered as high-quality studies and were therefore included in the meta-analysis.

\section{Statistical analysis}

We chose the HRs and ORs of the primary endpoints as the effect size between the statin and non-statin groups. The risk of death was significantly higher in the statin group than in the non-statin group if the mean effect size was greater than 1 and if the calculated $95 \%$ CI did not include 1.

The selection of a fixed effects model or a random effects model for the calculation of the average effect size was decided assuming that the studies were derived from the same population and the analyses had the same purpose. These assumptions were considered according to the characteristics of the study, i.e., the study subjects, intervention methods, and study environment. The Cochrane chi-square test and I statistics were used to determine heterogeneity between the studies for metaanalysis. In particular, the fixed effects model was selected 


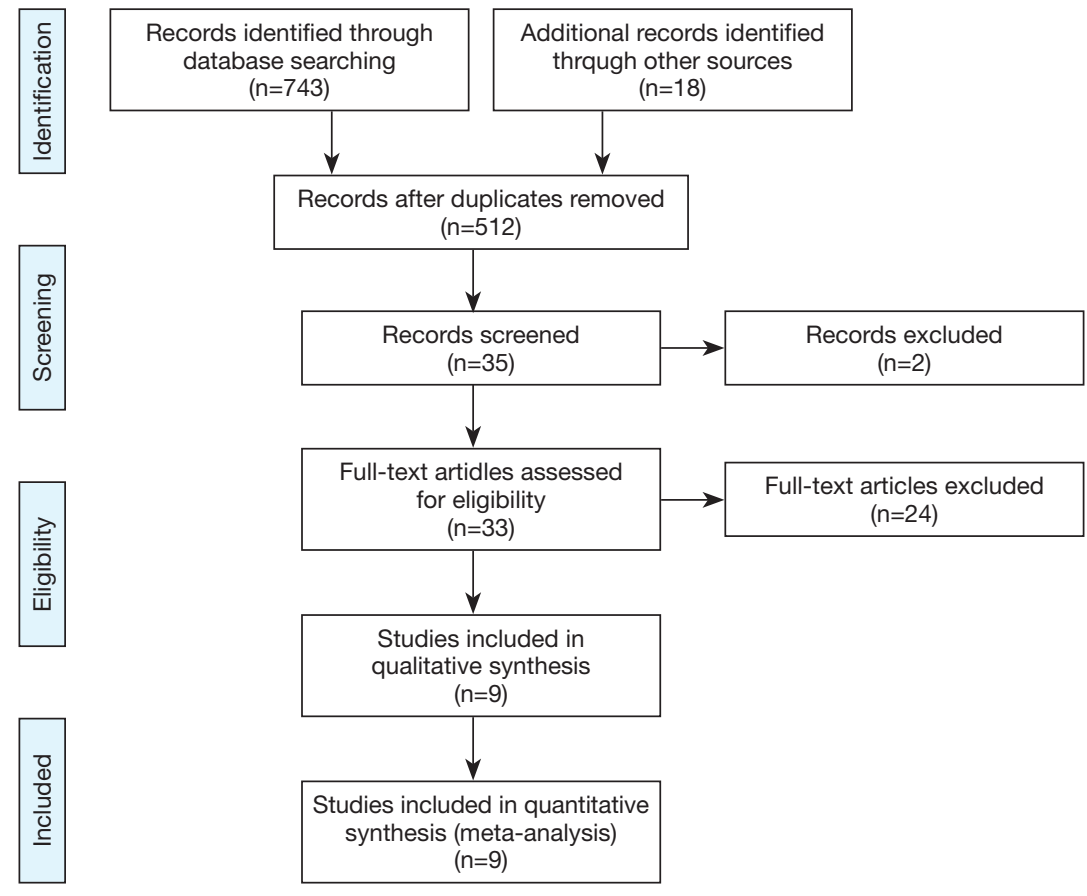

Figure 1 Flow chart of study selection.

if the homogeneity statistic $\left(I^{2}\right)$ was $<50 \%$ or the $\mathrm{P}$ value of the $Q$ statistic was $>0.05$. In all other cases, the random effects model was used. Begg's funnel plot and Egger's linear regression were used to assess for potential publication bias $(25,26)$. The funnel plots were visually assessed for asymmetry. In addition, as the quality of data of prospective cohort studies is better than that of retrospective cohort studies, a 2-fold weight was applied for prospective cohort studies, as a sensitivity analysis. All statistical analyses were performed by a statistician (S Choi) using $\mathrm{R}$ version 3.6.1 (R Foundation for Statistical Computing, Vienna, Austria) with the meta package in this environment.

\section{Results}

We initially identified 743 articles, of which 512 articles were selected after removing duplicated records. After screening by title and abstract, we identified 35 cohort studies that appeared to address issues potentially related to the primary study question. Next, after completely reading the 35 articles, 9 studies were included in the meta-analysis, as shown in Figure 1 (13-21). Six of the nine studies were included in the meta-analysis of 30-day mortality $(13,15$ $18,20)$, and 3 studies were included in the meta-analysis of in-hospital mortality $(14,19,21)$. As the 30 -day mortality and hospital mortality were presented as HRs with $95 \%$ CIs in six studies $(13,15-18,20)$ and as ORs with $95 \%$ CIs in three studies $(14,19,21)$, HRs and ORs were used as endpoints for 30-day mortality and hospital mortality, respectively, in this meta-analysis. In addition, we had the full raw data for the study published by Oh et al. (15), which was conducted recently at our institution, and the HRs for 30-day mortality were re-analyzed to include the outcomes in this meta-analysis. The characteristics of the cohort studies are presented in Table 1, and the points allocated according to the Newcastle Ottawa Scale are presented in Supplementary Table S1. All nine studies scored relatively high scores of 8-9 points on the Newcastle Ottawa Scale, so the nine studies were not excluded. Finally, a total of 199,985 critically ill patients from nine studies (44,582 and 155,403 in the statin and non-statin groups, respectively) were included in the meta-analysis.

\section{0-day mortality}

Figure 2 shows the results of the meta-analysis of 30-day mortality among statin users compared with non-statin users before (A) and after (B) applying a 2-fold weight to prospective cohort studies. The analysis of heterogeneity among the six studies yielded an $I^{2}$ value of $94 \%(\mathrm{P}<0.01)$, 


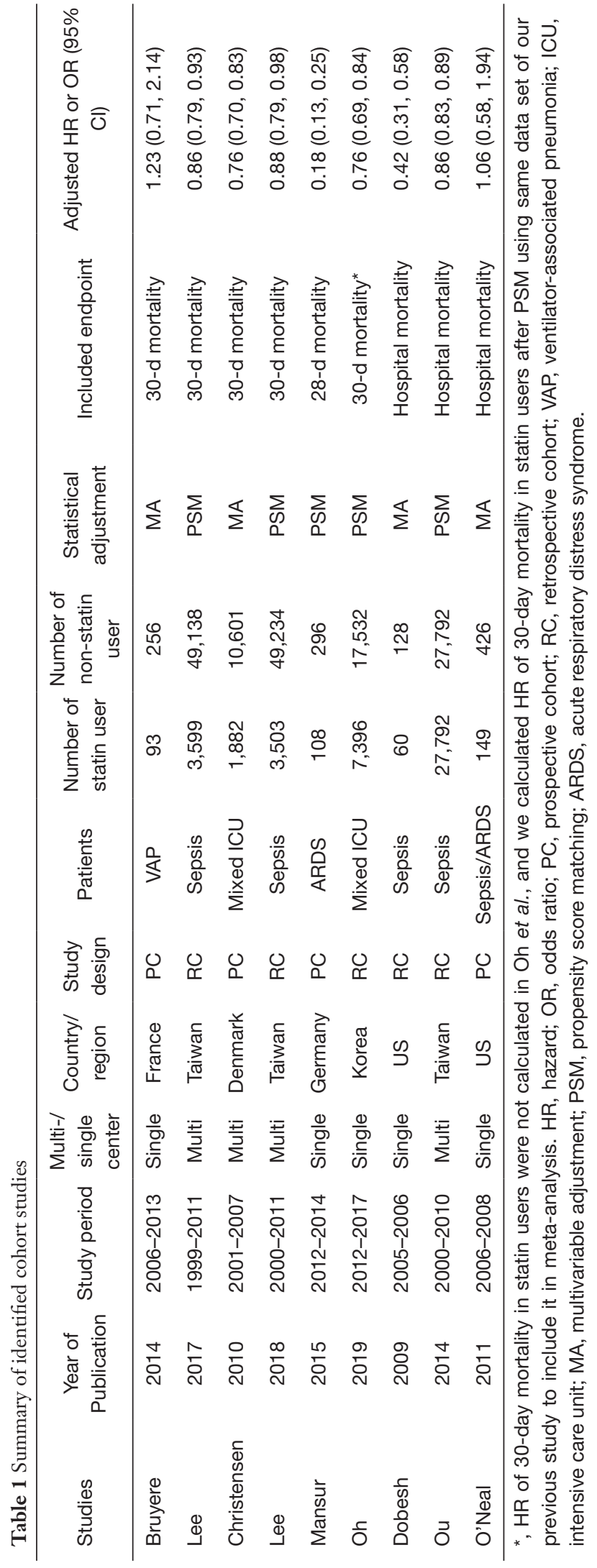

indicating significant variations among the studies. The results of the random effects model showed that the 30-day mortality rate among statin users was $31 \%$ lower than that among non-statin users (HR $=0.69,95 \%$ CI: 0.56-0.85). After applying the 2 -fold weight, the 30 -day mortality rate among statin users was $34 \%$ lower than that among nonstatin users (HR $=0.66$, 95\% CI: 0.46-0.95). The funnel plot indicates that there was no publication bias ( $\mathrm{P}$ value of Egger's test $=0.338$; Figure S1).

Figure 3 shows the results of meta-analysis of 30-day mortality among atorvastatin (A) and simvastatin (B) users compared with non-statin users. In the fixed effects models, the 30-day mortality rate among both atorvastatin users (HR $=0.78,95 \%$ CI: $0.72-0.84$ ) and simvastatin users (HR $=0.84$, 95\% CI: 0.78-0.91) was lower than that among non-statin users.

\section{In-bospital mortality}

Figure 4 shows the results of the meta-analysis of in-hospital mortality among statin users compared with non-statin users. The analysis of heterogeneity among the three studies yielded an $I^{2}$ value of $90 \%(\mathrm{P}<0.01)$, indicating significant variations among the studies. Therefore, the results of the random effects model revealed that compared with no statin use, statin use was not significantly associated with inhospital mortality (OR $=0.71,95 \%$ CI: $0.42-1.21$ ).

\section{Discussion}

In this systemic review and meta-analysis of cohort studies, we showed that there was a significant association of prior statin therapy with 30-day mortality rate among critically ill patients, whereas there was no significant association of prior statin therapy with in-hospital mortality. In addition to the results of a previous meta-analysis of RCTs (12), which focused on the effect of statin therapy after ICU admission or diagnosis of a specific disease (sepsis or ARDS), our results suggest that statin therapy before ICU admission might have a potential benefit in terms of 30-day mortality among critically ill patients.

The most important characteristic of our study is that we only included cohort studies that focused on the effect of prior statin therapy in critically ill patients. Long-term statin exposure is known to cause immuno-modulation by a pleiotropic effect (27), and it suggested that prior statin therapy before ICU admission or diagnosis of a specific disease, such as sepsis or ARDS, might affect patient 
A 30d mortality: before weighting

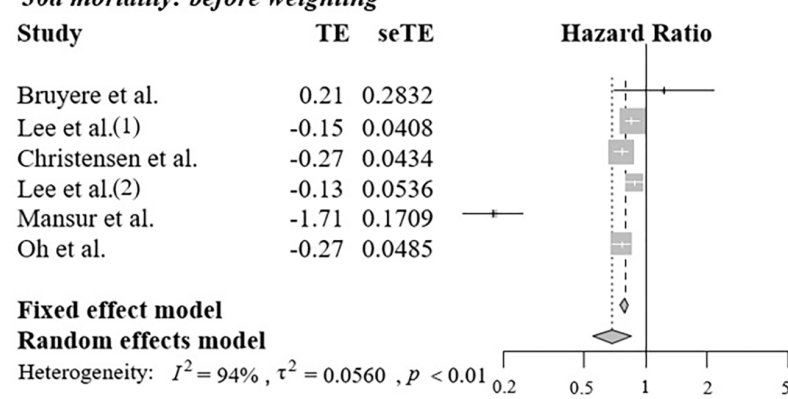

B 30d mortality: after weighting

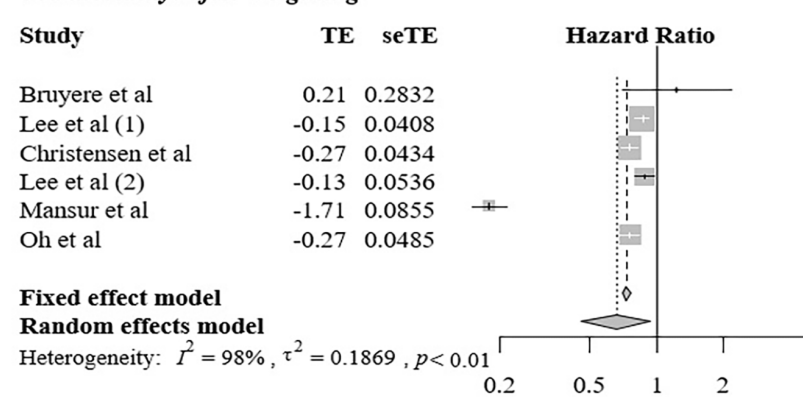

HR $\quad 95 \% \mathrm{CI}$ Weight Weight

$1.23[0.71 ; 2.14] \quad 0.6 \% \quad 8.4 \%$

$0.86[0.79 ; 0.93] \quad 30.7 \% \quad 19.7 \%$

$0.76[0.70 ; 0.83] \quad 27.2 \% \quad 19.7 \%$

$0.88[0.79 ; 0.98] \quad 17.8 \% \quad 19.3 \%$

$0.18[0.13 ; 0.25] \quad 1.8 \% \quad 13.4 \%$

$0.76[0.69 ; 0.84] \quad 21.8 \% \quad 19.5 \%$

$0.79[0.76 ; 0.83] \quad 100.0 \% \quad-$

$0.69[0.56 ; 0.85] \quad-\quad 100.0 \%$

Weight Weight

(fixed) (random)

$1.23[0.71 ; 2.14] \quad 0.6 \% \quad 12.5 \%$

$0.86[0.79 ; 0.93] \quad 29.2 \% \quad 17.6 \%$

$0.76[0.70 ; 0.83] \quad 25.9 \% \quad 17.6 \%$

$0.88[0.79 ; 0.98] \quad 17.0 \% \quad 17.5 \%$

$0.18[0.15 ; 0.21] \quad 6.7 \% \quad 17.1 \%$

$0.76[0.69 ; 0.84] \quad 20.7 \% \quad 17.6 \%$

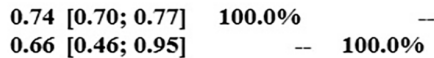

Figure 2 Meta-analysis of 30-day mortality among statin users compared with non-statin users before (A) and after (B) applying a 2-fold weight to prospective cohort studies.

A 30d mortality: Atorvastatin

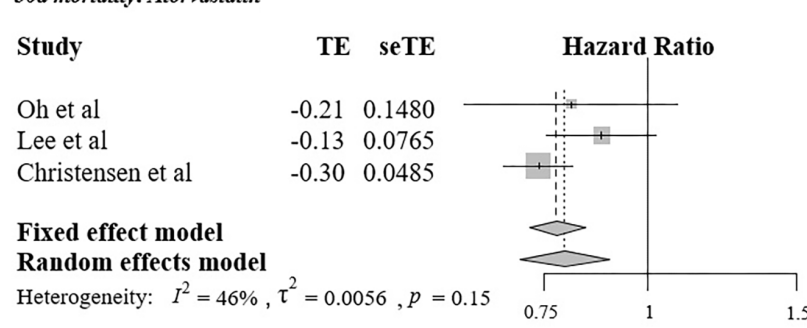

B

$\begin{array}{lrr}\begin{array}{lrl}\text { 30d mortality: Simvastatin } \\ \text { Study }\end{array} & \text { TE } & \text { seTE } \\ & & \\ \text { Oh et al. } & -0.22 & 0.0587 \\ \text { Lee et al. } & -0.15 & 0.0510 \\ \text { Christensen et al. } & -0.05 & 0.1352 \\ & & \\ \text { Fixed effect model } & \\ \text { Random effects model } & \\ \text { Heterogeneity: } I^{2}=0 \%, \tau^{2}=0, p=0.42\end{array}$

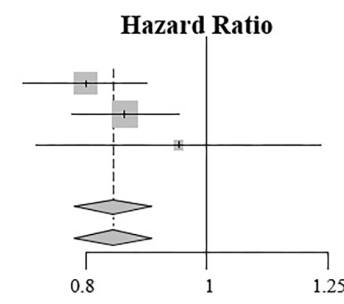

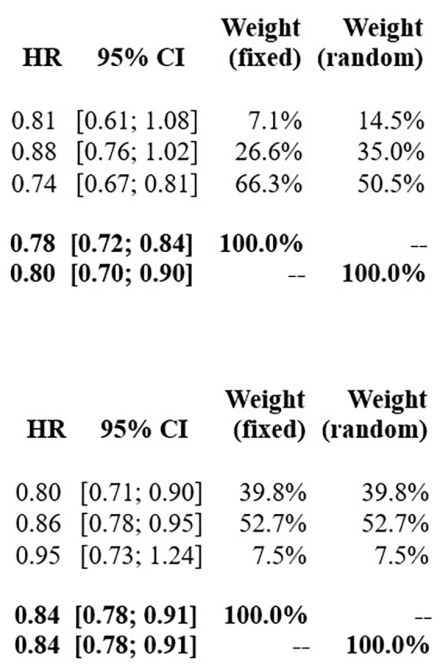

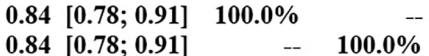

Figure 3 Meta-analysis of 30-day mortality among atorvastatin users (A) and simvastatin users (B) compared with non-statin users.

prognosis. However, it might be impossible to demonstrate this in a RCT because the development of sepsis or ARDS and ICU admission are not usually predictable and thus statin exposure before the events cannot be evaluated in a retrospective cohort design. Our meta-analysis included a prospective cohort study (18) that prospectively included patients with sepsis-associated ARDS, but it evaluated the history of prior statin therapy retrospectively, in addition to the effect of statin therapy after the diagnosis of sepsis-associated ARDS. A recent meta-analysis of five 


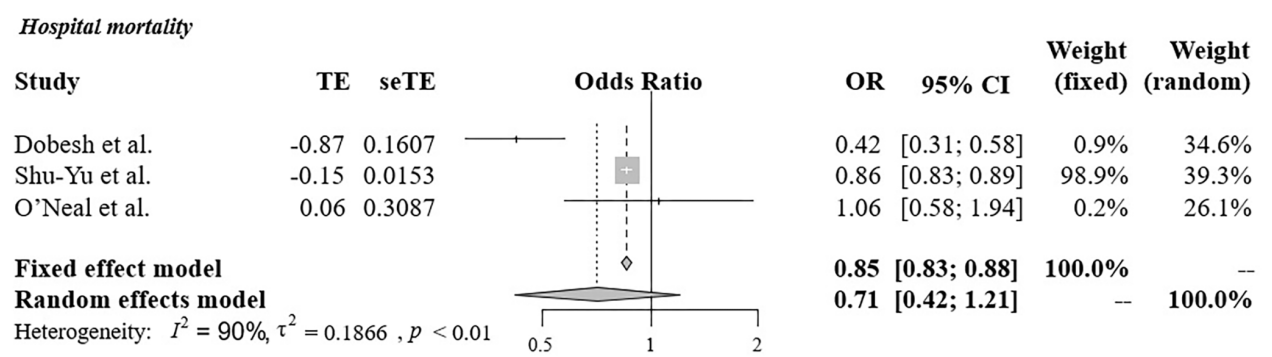

Figure 4 Meta-analysis of in-hospital mortality among statin users compared with non-statin users.

retrospective cohort studies, with a similar design to our meta-analysis, reported that metformin exposure before ICU admission was associated with a lower mortality in adult patients with diabetes mellitus diagnosed with sepsis (28). Therefore, our rationale behind inclusion of cohort studies should be interpreted in consideration of these perspectives.

While a previous meta-analysis of RCTs concluded that there was no significant effect of statin therapy in sepsis patients (12), we found a significant association of prior statin therapy with mortality among critically ill patients. Our results should be interpreted carefully considering the characteristics of the cohort studies. RCTs and populationbased cohort studies have different strengths and limitations (29) and might arrive at different conclusions for several reasons (30). First, cohort studies may have a more pronounced selection bias or more confounders because of the absence of randomization. Second, the generalizability of the results of RCTs and cohort studies might be different because many RCTs have specific inclusion and exclusion criteria that are often restrictive, whereas cohort studies usually include much broader populations (31). Third, as RCTs may sometimes be underpowered for detecting important differences in outcomes, they can lead to erroneous conclusions (generally false negatives). The total sample size of this meta-analysis was sufficiently large (with 199,985 patients from nine studies); therefore, it was possible to detect a beneficial association of prior statin therapy with mortality with adequate statistical power rather than to perform a meta-analysis of RCTs.

The diverse characteristics in this meta-analysis should also be considered when interpreting the results of this study. In this meta-analysis, we defined and included "critically ill patients" as those who required treatment or monitoring in the ICU. Therefore, the mixed ICU population, sepsis patients, ARDS patients, and patients with VAP were all included in this study. The World Federation of Societies of Intensive and Critical Care Medicine convened a task force to define ICU. They defined ICU as a health care resource that provides intensive and specialized medical and nursing care to critically ill patients (32). From this perspective, our broader inclusion criteria of critically ill patients in this meta-analysis can be understood.

This study has some limitations. First, the nine studies included in the meta-analysis did not use identical statistical methods to adjust for confounders. Also, the number of confounders, diseases, and types of confounders considered in each study were not identical. This is a common limitation of all meta-analyses; a random effects model had to be used to adjust for the differences. Third, five retrospective cohort studies were included in this metaanalysis. Compared with prospective cohort studies, the quality of data in retrospective cohort studies is not guaranteed, which might in turn affect the results of the meta-analysis. Fourth, only three studies were included to evaluate the effect of prior statin therapy on in-hospital mortality among critically ill patients; thus, the results regarding in-hospital mortality should be interpreted carefully. Fifth, we did not evaluate the effect of dosage among prior statin users in this meta-analysis because of the lack of data. Finally, as mentioned above, our study broadly included critically ill patients; therefore, our results might not be generalizable to specific disease groups, such as patients with sepsis or ARDS.

In conclusion, this study showed that there was a beneficial association of prior statin therapy with 30-day mortality among critically ill patients. However, there was no significant association of prior statin therapy with inhospital mortality. Future prospective cohort studies with large sample sizes are required to confirm our findings. 


\section{Acknowledgments}

Funding: None.

\section{Footnote}

Conflicts of Interest: The authors have no conflicts of interest to declare.

Ethical Statement: The authors are accountable for all aspects of the work in ensuring that questions related to the accuracy or integrity of any part of the work are appropriately investigated and resolved. This study was approved by the Institutional Review Board of SNUBH (IRB approval number: X-1907/555-905), which waived the need for informed consent because of the retrospective nature of this meta-analysis.

Open Access Statement: This is an Open Access article distributed in accordance with the Creative Commons Attribution-NonCommercial-NoDerivs 4.0 International License (CC BY-NC-ND 4.0), which permits the noncommercial replication and distribution of the article with the strict proviso that no changes or edits are made and the original work is properly cited (including links to both the formal publication through the relevant DOI and the license). See: https://creativecommons.org/licenses/by-nc-nd/4.0/.

\section{References}

1. Santodomingo-Garzón T, Cunha TM, Verri WA Jr, et al. Atorvastatin inhibits inflammatory hypernociception. Br J Pharmacol 2006;149:14-22.

2. Rosenson RS. Low high-density lipoprotein cholesterol and cardiovascular disease: risk reduction with statin therapy. Am Heart J 2006;151:556-63.

3. Novack V, Terblanche M, Almog Y. Do statins have a role in preventing or treating sepsis? Crit Care 2006;10:113.

4. Terblanche M, Almog Y, Rosenson RS, et al. Statins and sepsis: multiple modifications at multiple levels. Lancet Infect Dis 2007;7:358-68.

5. Wang CY, Liu PY, Liao JK. Pleiotropic effects of statin therapy: molecular mechanisms and clinical results. Trends Mol Med 2008;14:37-44.

6. Mihos CG, Salas MJ, Santana O. The pleiotropic effects of the hydroxy-methyl-glutaryl-CoA reductase inhibitors in cardiovascular disease: a comprehensive review. Cardiol Rev 2010;18:298-304.
7. Janda S, Young A, Fitzgerald JM, et al. The effect of statins on mortality from severe infections and sepsis: a systematic review and meta-analysis. J Crit Care 2010;25:656.e7-22.

8. Deshpande A, Pasupuleti V, Rothberg MB. Statin therapy and mortality from sepsis: a meta-analysis of randomized trials. Am J Med 2015;128:410-7.e1.

9. Kruger P, Bailey M, Bellomo R, et al. A multicenter randomized trial of atorvastatin therapy in intensive care patients with severe sepsis. Am J Respir Crit Care Med 2013;187:743-50.

10. Kruger PS, Harward ML, Jones MA, et al. Continuation of statin therapy in patients with presumed infection: a randomized controlled trial. Am J Respir Crit Care Med 2011;183:774-81.

11. Singh RK, Agarwal V, Baronia AK, et al. The Effects of Atorvastatin on Inflammatory Responses and Mortality in Septic Shock: A Single-center, Randomized Controlled Trial. Indian J Crit Care Med 2017;21:646-54.

12. Chen M, Ji M, Si X. The effects of statin therapy on mortality in patients with sepsis: A meta-analysis of randomized trials. Medicine (Baltimore) 2018;97:e11578.

13. Christensen S, Thomsen RW, Johansen MB, et al. Preadmission statin use and one-year mortality among patients in intensive care - a cohort study. Crit Care 2010;14:R29.

14. Dobesh PP, Klepser DG, McGuire TR, et al. Reduction in mortality associated with statin therapy in patients with severe sepsis. Pharmacotherapy 2009;29:621-30.

15. Oh TK, Song IA, Lee JH, et al. Preadmission Statin Use and 90-day Mortality in the Critically Ill: A Retrospective Association Study. Anesthesiology 2019;131:315-27.

16. Lee CC, Lee MG, Hsu TC, et al. A Population-Based Cohort Study on the Drug-Specific Effect of Statins on Sepsis Outcome. Chest 2018;153:805-15.

17. Lee MG, Lee CC, Lai CC, et al. Preadmission statin use improves the outcome of less severe sepsis patients - a population-based propensity score matched cohort study. Br J Anaesth 2017;119:645-54.

18. Mansur A, Steinau M, Popov AF, et al. Impact of statin therapy on mortality in patients with sepsis-associated acute respiratory distress syndrome (ARDS) depends on ARDS severity: a prospective observational cohort study. BMC Med 2015;13:128.

19. Ou SY, Chu H, Chao PW, et al. Effect of the use of low and high potency statins and sepsis outcomes. Intensive Care Med 2014;40:1509-17.

20. Bruyere R, Vigneron C, Prin S, et al. Impact of prior statin therapy on the outcome of patients with suspected 
ventilator-associated pneumonia: an observational study. Crit Care 2014;18:R83.

21. O’Neal HR Jr, Koyama T, Koehler EA, et al. Prehospital statin and aspirin use and the prevalence of severe sepsis and acute lung injury/acute respiratory distress syndrome. Crit Care Med 2011;39:1343-50.

22. Stroup DF, Berlin JA, Morton SC, et al. Meta-analysis of observational studies in epidemiology: a proposal for reporting. Meta-analysis Of Observational Studies in Epidemiology (MOOSE) group. JAMA 2000;283:2008-12.

23. Moher D, Liberati A, Tetzlaff J, et al. Preferred reporting items for systematic reviews and meta-analyses: the PRISMA statement. BMJ 2009;339:b2535.

24. Stang A. Critical evaluation of the Newcastle-Ottawa scale for the assessment of the quality of nonrandomized studies in meta-analyses. Eur J Epidemiol 2010;25:603-5.

25. Begg CB, Mazumdar M. Operating characteristics of a rank correlation test for publication bias. Biometrics 1994;50:1088-101.

26. Stuck AE, Rubenstein LZ, Wieland D. Bias in metaanalysis detected by a simple, graphical test. Asymmetry detected in funnel plot was probably due to true

Cite this article as: Oh TK, Song IA, Choi S. Prior statin therapy and mortality among critically ill patients: a systemic review and meta-analysis of cohort studies. Ann Transl Med 2020;8(6):396. doi: 10.21037/atm.2020.02.101 heterogeneity. Br Med J 1998;316:469.

27. Palinski $W$, Tsimikas S. Immunomodulatory effects of statins: mechanisms and potential impact on arteriosclerosis. J Am Soc Nephrol 2002;13:1673-81.

28. Liang H, Ding X, Li L, et al. Association of preadmission metformin use and mortality in patients with sepsis and diabetes mellitus: a systematic review and meta-analysis of cohort studies. Crit Care 2019;23:50.

29. Booth CM, Tannock IF. Randomised controlled trials and population-based observational research: partners in the evolution of medical evidence. Br J Cancer 2014;110:551-5.

30. Hannan EL. Randomized clinical trials and observational studies: guidelines for assessing respective strengths and limitations. JACC Cardiovasc Interv 2008;1:211-7.

31. Sørensen HT, Lash TL, Rothman KJ. Beyond randomized controlled trials: a critical comparison of trials with nonrandomized studies. Hepatology 2006;44:1075-82.

32. Marshall JC, Bosco L, Adhikari NK, et al. What is an intensive care unit? A report of the task force of the World Federation of Societies of Intensive and Critical Care Medicine. J Crit Care 2017;37:270-6. 
Table S1 The Newcastle-Ottawa quality assessment scale of including studies

\begin{tabular}{|c|c|c|c|c|c|c|c|c|c|c|}
\hline \multirow[b]{2}{*}{ Studies } & \multicolumn{3}{|c|}{ Selection } & \multicolumn{3}{|c|}{ Comparability } & \multicolumn{3}{|c|}{ Assessment of outcome } & \multirow[b]{2}{*}{$\begin{array}{l}\text { Total quality } \\
\text { score }\end{array}$} \\
\hline & $\begin{array}{c}\text { Represent } \\
\text {-activeness of } \\
\text { exposure arm }\end{array}$ & $\begin{array}{l}\text { Selection of the } \\
\text { comparative arm }\end{array}$ & $\begin{array}{l}\text { Origin of } \\
\text { exposure } \\
\text { source }\end{array}$ & $\begin{array}{c}\text { Demonstration that } \\
\text { outcome of interest } \\
\text { was not present at } \\
\text { start of study }\end{array}$ & $\begin{array}{l}\text { Studies controlling } \\
\text { the most important } \\
\text { factors }\end{array}$ & $\begin{array}{l}\text { Studies controlling } \\
\text { the other main } \\
\text { factors }\end{array}$ & $\begin{array}{l}\text { Assessment of } \\
\text { outcome with } \\
\text { independency }\end{array}$ & $\begin{array}{l}\text { Adequacy of } \\
\text { follow-up length } \\
\text { (to assess } \\
\text { outcome) }\end{array}$ & $\begin{array}{l}\text { Lost to follow-up } \\
\text { acceptable (less } \\
\text { than } 10 \% \text { and } \\
\text { reported) }\end{array}$ & \\
\hline Bruyere & * & * & * & * & * & * & * & * & * & 9 \\
\hline Lee & * & * & * & * & * & * & * & * & * & 9 \\
\hline Christensen & * & * & * & * & * & * & * & * & * & 9 \\
\hline Lee & * & * & * & * & * & * & * & * & * & 9 \\
\hline Mansur & * & * & * & * & * & * & * & * & * & 9 \\
\hline Oh & * & * & * & * & * & * & * & * & * & 9 \\
\hline Dobesh & * & * & * & * & * & * & * & * & * & 9 \\
\hline $\mathrm{Ou}$ & * & * & * & * & * & * & * & * & * & 9 \\
\hline O'Neal & * & * & * & * & * & * & * & * & & 8 \\
\hline
\end{tabular}

*. 1 point.

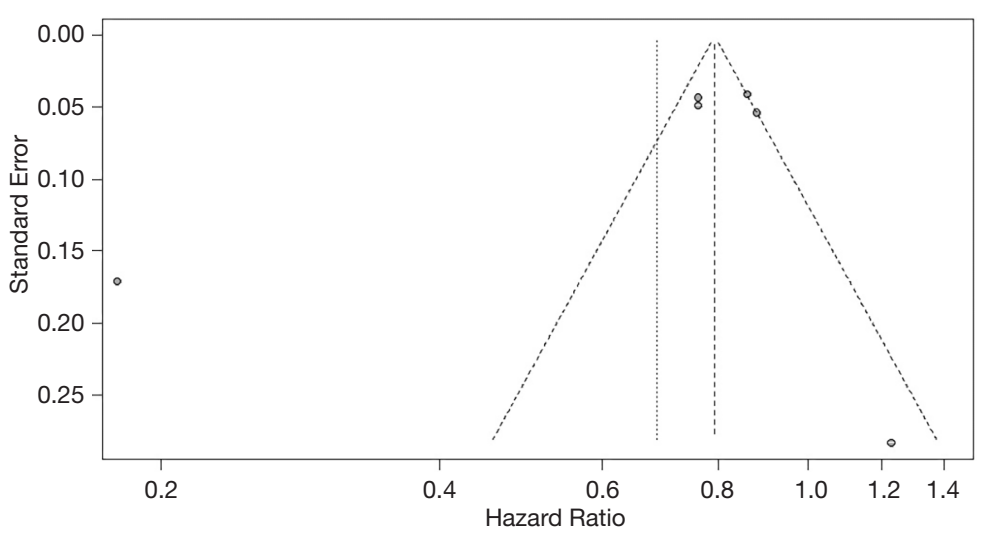

Figure S1 Begg's funnel plot. 